\title{
Methotrexate in the Treatment of Generalized Pustular Psoriasis with Liver Involvement: A Case Report
}

\author{
Houshmand $\mathbf{H}^{1}$, Nabavizadeh $\mathbf{H}^{2}$, Houshmand $\mathrm{G}^{3}$, Ghafouri $\mathrm{Z}^{4}$, \\ Alyasin $\mathrm{S}^{2}$ and Kouhsari $\mathrm{E}^{5,6 *}$ \\ ${ }^{1}$ Department of Pediatrics, Urmia University of Medical Science, Iran \\ 2Department of Pediatrics, Shiraz University of Medical Sciences, Iran \\ 3Department of Pharmacology, Mazandaran University of Medical Sciences, Iran \\ ${ }^{4}$ Department of Biochemistry Biophysics and Genetics, Mazandaran University of Medical Sciences, Iran \\ ${ }^{5}$ Clinical Microbiology Research Center, Ilam University of Medical Sciences, Iran \\ ${ }^{6}$ Laboratory Sciences Research Center, Golestan University of Medical Sciences, Iran
}

Case Report

Volume 3 Issue 1

Received Date: July 16, 2019

Published Date: July 30, 2019

*Corresponding author: Ebrahim Kouhsari, Clinical Microbiology Research Center, Ilam University of Medical Sciences, Ilam, Iran, PO Box: 6939177143, Tel/Fax: 08432227101; Email: Kouhsari-e@medilam.ac.ir

\section{Abstract}

Generalized pustular psoriasis is a dangerous medical problem with abrupt onset. Here, we describe a ten-month-old girl with GPP. The girl presented small sized erythema and pustules on her cheek, which extended to her abdomen, upper and lower extremities. Skin biopsy was in favor of GPP. Despite negative microbial cultures and viral markers, laboratory data showed abnormal liver enzymes. After admission, she responded well to local emollient agents, antihistamine, systemic antibiotic and oral Methotrexate. Our report is special because despite increase in patient's liver enzymes, Methotrexate, which is a liver complication potential drug, simultaneously improved general condition and liver enzymes.

Keywords: Seborrheic Dermatitis; Generalized Pustular Psoriasis; Methotrexate

Abbreviations: GPP: Generalized Pustular Psoriasis; MTX: Methotrexate; CBC: Complete Blood Count; LFT: Liver Function Test; ASO: Anti Streptolysine-0; PMN: Polymorphonuclear.

\section{Introduction}

Psoriasis is a common, chronic, painful, disfiguring, disabling, and non-communicable skin disease of variable severity that affects $1 \%$ to $2 \%$ of the general population and contributes about $4 \%$ of the childhood dermatoses
[1-3]. 125 million people worldwide, 2 to 3 percent of the total population have psoriasis, according to the World Psoriasis Day consortium [4]. Generalized pustular psoriasis (GPP) is a severe form of psoriasis, rare in the pediatric age group, a neutrophilic dermatosis, and commonly associated with life-threatening complications and various toxic, systemic symptoms (heart failure, renal failure, and sepsis) that can be challenging to treat [1,5]. GPP most affected children aged between 2 and 10 at the time of onset [6]. The commonly first-line treatments of GPP are methotrexate (MTX), ciclosporin, acitretin and 
etretinate [7]. The mechanism of action the MTX is inhibits dihydrofolate-reductase competitively and finally disturbed in synthesis of purin, thymin and DNA. Here, we report a case of GPP with Liver involvement successfully treated with MTX.

\section{Case Report}

The case we present is a ten-month-old girl with no history of skin disease in her family who developed pruritic erythematous papules on her cheeks 75 days prior to hospital admission. The lesions then progressed and appeared all over her trunk; later pustules appeared in clusters over the erythematous lesions, which were crusted 50 days after the onset of the disease. During her illness, she developed intermittent high-grade fever. Due to aggravation of her disease, she was admitted to hospital. On arrival, her physical examination revealed generalized erythematous plaques with overlying pustules, severe desquamation in her face, scalp, abdomen, legs and forearms, revealing GPP. At the time, she had no mucous membrane involvement. After one day her feet, hands and forearms also got involved. During admission, she was visited by several physicians and treated with intravenous antibiotics, local dressing with emollients, Mupirocin and Betamethasone ointment. The following days she developed facial edema and irritability (Figure 1).

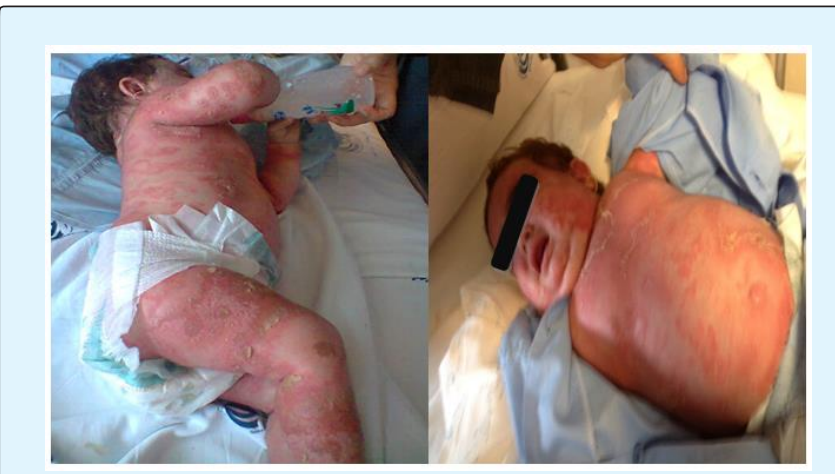

Figure 1: Facial edema and irritability.

A complete blood count (CBC), liver function test (LFT) and Anti Streptolysine-O (ASO) titer were done. Leukocytosis (White Blood Cell count, WBC 16500) with neutrophilia (85\%), mildly elevated liver enzymes (AST 85 unit/L, ALT 115 unit/L) and negative ASO titer (100 Todd units) were detected. Gram stain, culture and direct examination ( $\mathrm{KOH}$ smear) of skin lesions were also prepared which all resulted negative. ESR and CRP levels were $95 \mathrm{~mm} /$ hour and $105 \mathrm{mg} / \mathrm{dl}$, respectively. A $2.5 \mathrm{~mm}$ punch biopsy was taken from the skin lesions, which showed: parakeratosis, regular acanthosis with focal Neutrophil aggregate formation, absence of granular layer and polymorphonuclear (PMN) exocytosis, mild lymphocyte admixed with few PMN in papillary dermis. The pathologic finding was congruent with psoriasis (Figure 2a). Microscopic examination revealed regular acanthosis with absence of granular layer, confluent parakeratosis with accumulation of neutrophils (Munro abscess) and mild exocytosis of neutrophils. Papillary dermis showed edema and mild mixed inflammatory infiltrate composed of lymphocytes and neutrophils (Figure 2b).

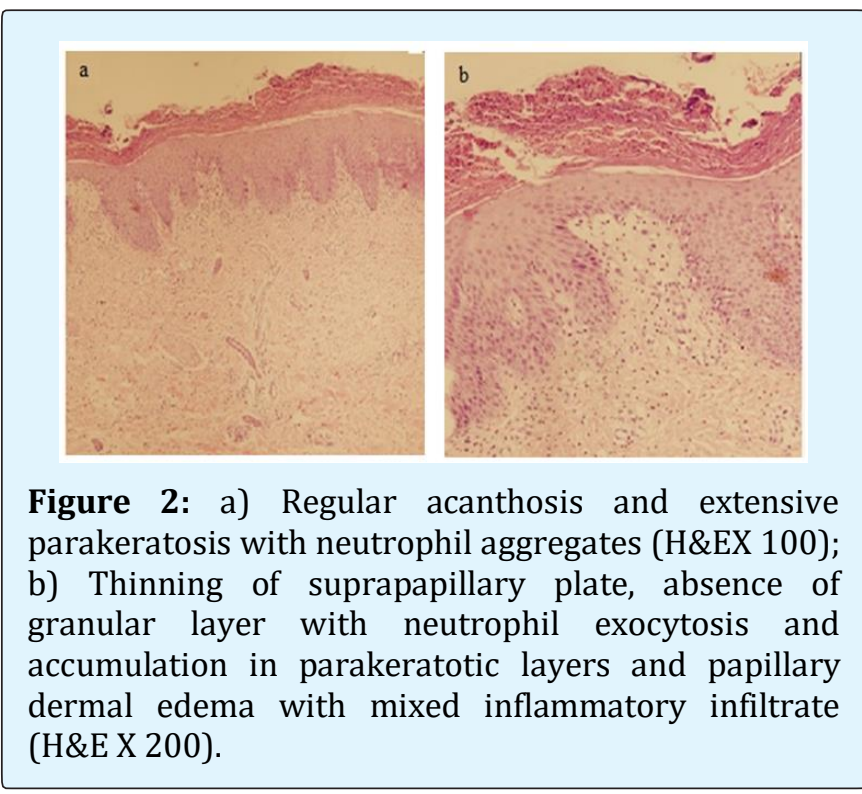

We did not perform liver biopsy for patient, because the procedure was invasive and no finding regarding cholestasis was seen, nor serious symptoms of liver failure. Other investigations such as ophthalmic examination showed no ocular involvement. Finally, a clinical diagnosis of psoriasis was made, and she was treated with oral MTX (once weekly in doses of 0.2 to 0.4 $\mathrm{mg} / \mathrm{kg}$ ), and oral Folic acid (daily at the dose of $1 \mathrm{mg}$ ). After 3 days, her skin lesions resolved and after a month, complete resolution of skin lesions and liver improvement were achieved.

\section{Discussion}

GPP is a serious global problem with a high global burden of disease. GPP is distinctive, in the way that it starts with localized burning erythema, which involves large areas and creates a fiery-red skin in hours [8]. On the erythematous skin, pinpoint pustules appear in clusters, becoming confluent and forming lakes filled with 


\section{Journal of Infectious Diseases \& Travel Medicine}

purulent fluid [9]. Differential diagnosis of GPP is; acute generalized exanthematous pustulosis, severe impetigo, extensive tinea corporis, infected generalized atopic dermatitis and or seborrheic dermatitis, fever, generalized malaise, leukocytosis and sub-corneal pustular dermatosis [10]. The complications of this disease are skin streptococcal or viral infections, increased susceptibility to sunburn, withdrawal symptoms of corticosteroid therapy [11]. Skin manifestations are associated with abnormal proliferation and differentiation of keratinocytes and infiltration of inflammatory cells to epidermis and dermis due to T-cell abnormality. Both genetic and environmental factors are involved in the pathogenesis of this disease. Mutations in IL-12 $\beta$, IL-23, IL-36 and HLA-class II alleles are the most compelling genetic factors involved in the pathogenesis of psoriasis $[12,13]$. Characteristic changes in skin biopsy of psoriatic lesions are leukocytosis, parakeratosis, acanthosis, elongated rete ridges, neutrophilic infiltrate in epidermis, and lymphocytic infiltration in the dermis.

Neutrophils may also invade mucosal and synovial membranes, resulting in poly-articular arthritis, uveitis, conjunctivitis and corneal ulceration. Moreover, there are reports declaring generalized pustular variant is sometimes associated with neutrophilic infiltration of liver, causing inflammation and destruction of hepatocytes [14]. Evidences show the involvement of other organs as well as skin in GPP; noninfectious acute respiratory distress syndrome (sterile pneumonitis) has been described. The pathogenesis of aseptic pneumonitis is unknown but various pro-inflammatory cytokines (TNF- $\alpha$ ) could play a role in the recruitment of leukocytes to the lung [15]. Viguier M, et al. [12] and Allez M, et al. [16] reported severe liver involvement and recurrent episodes of cholestatic jaundice could be associated with GPP. Neutrophilic cholangitis, neutrophilic infiltration in the skin, mucous membranes, portal tract, synovial membranes and ophthalmic involvement (conjunctivitis and uveitis) was observed in liver biopsy $[12,16]$. While our patient's liver issues were mild. Kaur I, et al. [2] reported that MTX is an effective, available, and reasonably safe drug to be used in severe childhood psoriasis under supervision and laboratory expert monitoring. Our patient's effective medication included MTX, as well.

\section{Conclusion}

This case illustrates with a diagnosis of GPP and an elevated liver enzyme was treated with MTX. This drug can cause liver cell damage. However, in our patient, this drug not only did not increase liver enzymes, but also it improved underlying liver complications. Thus, we conclude that MTX could help normalize the liver cell function in GPP, as well as the disease itself.

\section{References}

1. Jeon C, Nakamura M, Sekhon S, Yan D, Wu JJ, et al. (2017) Generalized pustular psoriasis treated with apremilast in a patient with multiple medical comorbidities. JAAD case reports 3(6): 495-497.

2. Kaur I, Dogra S, De D, Kanwar AJ (2008) Systemic methotrexate treatment in childhood psoriasis: further experience in 24 children from India. Pediatric Dermatology 25(2): 184-188.

3. WHO (2016) Global report on psoriasis: World Health Organization.

4. Consortium WPD (2013) Facts about psoriasis. Facts about Psoriasis Accessed 23.

5. de Moura CAGG, de Assis LH, Góes P, Rosa F, Nunes V, et al. (2015) A case of acute generalized pustular psoriasis of von zumbusch triggered by hypocalcemia. Case Rep Dermatol 7(3): 345-351.

6. Dogra S, Kumaran MS, Handa S, Kanwar AJ (2005) Methotrexate for generalized pustular psoriasis in a 2-year-old child. Pediatric Dermatology 22(1): 85-86.

7. Polesie S, Lidholm AG (2017) Secukinumab in the treatment of generalized pustular psoriasis: A case report. Acta Dermato Venereologica 97(1): 124-125.

8. Hoegler KM, John AM, Handler MZ, Schwartz RA (2018) Generalized pustular psoriasis: A review and update on treatment. J Eur Acad Dermatol Venereol 32(10): 1645-1651.

9. Zhou LL, Georgakopoulos JR, Ighani A, Yeung J (2018) Systemic monotherapy treatments for generalized pustular psoriasis: A systematic review. J Cutan Med Surg 22(6): 591-601.

10. Bejarano JJR, Valdecantos WC (2013) Psoriasis as autoinflammatory disease. Dermatologic clinics 31(3): 445-460.

11. Conway R, Low C, Coughlan RJ, O’Donnell MJ, Carey JJ (2015) Methotrexate use and risk of lung disease in psoriasis, psoriatic arthritis, and inflammatory bowel disease: Systematic literature review and metaanalysis of randomised controlled trials. BMJ 350: 1269. 


\section{Journal of Infectious Diseases \& Travel Medicine}

12. Viguier $M$, Allez $M$, Zagdanski AM, Bertheau $P$, de Kerviler E, et al. (2004) High frequency of cholestasis in generalized pustular psoriasis: Evidence for neutrophilic involvement of the biliary tract. Hepatology 40(2): 452-458.

13. Arakawa A, Vollmer S, Besgen P, Galinski A, Summer B, et al. (2018) Unopposed IL-36 activity promotes clonal CD4+ $\mathrm{T}$-cell responses with IL-17A production in generalized pustular psoriasis 138(6): 1338-1347.
14. Saikaly SK, Mattes M (2016) Biologics and pediatric generalized pustular psoriasis: An emerging therapeutic trend. Cureus 8(6): 652.

15. Katz M, Seidenbaum M, Weinrauch L (1987) Penicillin-induced generalized pustular psoriasis. J Am Acad Dermatol 17(5): 918-920.

16. Allez M, Roux ME, Bertheau P, Erlinger S, Degott C, et al. (2000) Recurrent cholestatic jaundice associated with generalized pustular psoriasis: evidence for a neutrophilic cholangitis. J Hepatol 33(1): 160-162. 